\title{
Offensive alliances in cubic graphs
}

\author{
J. A. Rodríguez* \\ Department of Computer Engineering and Mathematics \\ Rovira i Virgili University of Tarragona \\ Av. Països Catalans 26, 43007 Tarragona, Spain \\ J. M. Sigarreta ${ }^{\dagger}$ \\ Department of Mathematics \\ Carlos III University of Madrid \\ Avda. de la Universidad 30, 28911 Leganés (Madrid), Spain
}

\begin{abstract}
An offensive alliance in a graph $\Gamma=(V, E)$ is a set of vertices $S \subset V$ where for every vertex $v$ in its boundary it holds that the majority of vertices in $v$ 's closed neighborhood are in $S$. In the case of strong offensive alliance, strict majority is required. An alliance $S$ is called global if it affects every vertex in $V \backslash S$, that is, $S$ is a dominating set of $\Gamma$. The global offensive alliance number $\gamma_{o}(\Gamma)$ (respectively, global strong offensive alliance number $\left.\gamma_{\hat{o}}(\Gamma)\right)$ is the minimum cardinality of a global offensive (respectively, global strong offensive) alliance in $\Gamma$. If $\Gamma$ has global independent offensive alliances, then the global independent offensive alliance number $\gamma_{i}(\Gamma)$ is the minimum cardinality among all independent global offensive alliances of $\Gamma$. In this paper we study mathematical properties of the global (strong) alliance number of cubic graphs. For instance, we show that for all connected cubic graph of order $n$,

$$
\frac{2 n}{5} \leq \gamma_{i}(\Gamma) \leq \frac{n}{2} \leq \gamma_{\hat{o}}(\Gamma) \leq \frac{3 n}{4} \leq \gamma_{\hat{o}}(\mathcal{L}(\Gamma))=\gamma_{o}(\mathcal{L}(\Gamma)) \leq n,
$$
\end{abstract}

*e-mail:juanalberto.rodriguez@urv.net

$\dagger$ †-mail:josemaria.sigarreta@uc3m.es 
where $\mathcal{L}(\Gamma)$ denotes the line graph of $\Gamma$. All the above bounds are tight.

Mathematics Subject Classification: 05C69; 15C05

Keywords: offensive alliance, global alliance, domination, independence number, cubic graphs.

\section{Introduction}

The study of defensive alliances in graphs, together with a variety of other kinds of alliances, was introduced in [6]. In the cited paper there was initiated the study of the mathematical properties of alliances. In particular, several bounds on the defensive alliance number were given. The particular case of global (strong) defensive alliance was investigated in [5] where several bounds on the global (strong) defensive alliance number were obtained. In [7] there were obtained several tight bounds on different types of alliance numbers of a graph, namely the (global) defensive alliance number, (global) offensive alliance number and (global) dual alliance number. In particular, there was investigated the relationship between the alliance numbers of a graph and its algebraic connectivity, its spectral radius, and its Laplacian spectral radius. A particular study of the alliance numbers, for the case of planar graphs, can be found in [8]. For the study of offensive alliances we cite [3, 4, 13] and for the study of alliances in trees we cite $[2,3,8]$. For the study of alliance free sets and alliance cover sets we cite $[10,11,9]$ and, finally, for the study of defensive alliances in the line graph of a simple graph we cite [12]. The aim of this work is to study global offensive alliances in cubic graphs.

\section{Preliminary Notes}

In this paper $\Gamma=(V, E)$ denotes a simple and connected graph of order $n$ and $\mathcal{L}(\Gamma)$ denotes the line graph of $\Gamma$. The degree of a vertex $v \in V$ will be denoted by $\delta(v)$ and the subgraph induced by a set $S \subset V$ will be denoted by $\langle S\rangle$. For a non-empty subset $S \subset V$, and a vertex $v \in V$, we denote by $N_{S}(v)$ the set of neighbors $v$ has in $S: N_{S}(v):=\{u \in S: u \sim v\}$. Similarly, we denote by $N_{V \backslash S}(v)$ the set of neighbors $v$ has in $V \backslash S: N_{V \backslash S}(v):=\{u \in V \backslash S: u \sim v\}$. 
The boundary of a set $S \subset V$ is defined as $\partial(S):=\bigcup_{v \in S} N_{V \backslash S}(v)$.

A non-empty set of vertices $S \subset V$ is called offensive alliance if and only if for every $v \in \partial(S),\left|N_{S}(v)\right| \geq\left|N_{V \backslash S}(v)\right|+1$. That is, a non-empty set of vertices $S \subset V$ is called offensive alliance if and only if for every $v \in \partial(S)$, $2\left|N_{S}(v)\right| \geq \delta(v)+1$.

An offensive alliance $S$ is called strong if for every vertex $v \in \partial(S)$, $\left|N_{S}(v)\right| \geq\left|N_{V \backslash S}(v)\right|+2$. In other words, an offensive alliance $S$ is called strong if for every vertex $v \in \partial(S), 2\left|N_{S}(v)\right| \geq \delta(v)+2$.

The offensive alliance number (respectively, strong offensive alliance number), denoted $a_{o}(\Gamma)$ (respectively, $a_{\hat{o}}(\Gamma)$ ), is defined as the minimum cardinality of an offensive alliance (respectively, strong offensive alliance) in $\Gamma$.

A non-empty set of vertices $S \subset V$ is a global offensive alliance if for every vertex $v \in V \backslash S,\left|N_{S}(v)\right| \geq\left|N_{V \backslash S}(v)\right|+1$. Thus, global offensive alliances are also dominating sets, and one can define the global offensive alliance number, denoted $\gamma_{o}(\Gamma)$, to equal the minimum cardinality of a global offensive alliance in $\Gamma$. Analogously, $S \subset V$ is a global strong offensive alliance if for every vertex $v \in V \backslash S,\left|N_{S}(v)\right| \geq\left|N_{V \backslash S}(v)\right|+2$, and the global strong offensive alliance number, denoted $\gamma_{\hat{o}}(\Gamma)$, is defined as the minimum cardinality of a global strong offensive alliance in $\Gamma$.

The independence number $\alpha(\Gamma)$ is the cardinality of the largest independent set of $\Gamma$. A set is an independent offensive alliance in $\Gamma$ if it is an offensive alliance and it is an independent set. If $\Gamma$ has independent offensive alliances, then the independent offensive alliance number $a_{i}(\Gamma)$ is the minimum cardinality among all independent offensive alliances of $\Gamma$. The domination number of $\Gamma$, denoted $\gamma(\Gamma)$, is the minimum cardinality of a dominating set in $\Gamma$. The independent domination number $i(\Gamma)$ is the minimum cardinality among all independent dominating sets of $\Gamma$. If $\Gamma$ has global independent offensive alliances, then the global independent offensive alliance number $\gamma_{i}(\Gamma)$ is the minimum cardinality among all independent global offensive alliances of $\Gamma$. Thus, $\gamma(\Gamma) \leq \gamma_{o}(\Gamma) \leq \gamma_{i}(\Gamma) \leq \alpha(\Gamma), a_{i}(\Gamma) \leq \gamma_{i}(\Gamma)$ and $i(\Gamma) \leq \gamma_{i}(\Gamma)$.

In this paper we study mathematical properties of the global (strong) alliance number of cubic graphs. For instance, we show that for all connected cubic graph of order $n$,

$$
\frac{2 n}{5} \leq \gamma_{i}(\Gamma) \leq \frac{n}{2} \leq \gamma_{\hat{o}}(\Gamma) \leq \frac{3 n}{4} \leq \gamma_{\hat{o}}(\mathcal{L}(\Gamma))=\gamma_{o}(\mathcal{L}(\Gamma)) \leq n .
$$

All the above bounds are tight. 


\section{Main Results}

These are the main results of the paper.

Theorem 3.1. Let $\Gamma$ be a connected cubic graph of order $n$.

1. $\frac{n}{2} \leq \gamma_{\hat{o}}(\Gamma) \leq \frac{3 n}{4}$.

2. $\gamma_{\hat{o}}(\Gamma)=\frac{n}{2}$ if and only if $\Gamma$ is a bipartite graph.

3. $\gamma_{\hat{o}}(\Gamma)=\frac{3 n}{4}$ if and only if $\Gamma$ is isomorphic to the complete graph $K_{4}$.

Proof.

1. If $\Gamma=(V, E)$ is $\delta$-regular, then $S \subset V$ is a $\delta$-dominating set if and only if $V \backslash S$ is an independent set. Therefore,

$$
\gamma_{\delta}(\Gamma)+\alpha(\Gamma)=n
$$

Moreover, if $\Gamma$ is a cubic graph, a set $S \subset V$ is a strong offensive alliance if and only if $S$ is a 3 -dominating set. Therefore,

$$
\gamma_{3}(\Gamma)=\gamma_{\hat{o}}(\Gamma)=n-\alpha(\Gamma)
$$

Finally, as for all $\delta$-regular graph $\Gamma, \alpha(\Gamma) \leq \frac{n}{2}$, the bound follows.

On the other hand, for all global strong offensive alliance $S$ such that $|S|=\gamma_{\hat{0}}(\Gamma), V \backslash S$ is an independent set. Thus, $\frac{3 n}{2} \leq 3\left(n-\gamma_{\hat{0}}(\Gamma)\right)+$ $\gamma_{\hat{0}}(\Gamma)$. Hence, the upper bound follows.

2. If $\Gamma=(V, E)$ is a bipartite cubic graph, then each set of the bipartition of $V$ is a strong global offensive alliance in $\Gamma$ of cardinality $\frac{n}{2}$, so $\left.\gamma_{\hat{0}}(\Gamma)\right)=\frac{n}{2}$. Conversely, if $S$ is a 3 -dominating set of cardinality $\frac{n}{2}$ in a cubic graph, then the edge-cut between $S$ and $V \backslash S$ has size $3 \frac{n}{2}$. Hence, both, $S$ and $V \backslash S$, are independent sets, so $\Gamma$ is a bipartite graph.

3. We only need to show that $\alpha(\Gamma)=\frac{n}{4} \Rightarrow \Gamma \cong K_{4}$. Suppose $\Gamma ¥ K_{4}$. If $X$ is an independent set such that $|X|=\alpha(\Gamma)=\frac{n}{4}$, then $\langle V \backslash X\rangle$ is the disjoin union of cycles. Let $x_{i}$ be the number of cycles of length $i$ in $V \backslash X, i=3, \ldots, \frac{3 n}{4}$. Thus,

$$
\sum i x_{i}=\frac{3 n}{4}
$$


If $x_{i}>0$, for some $i>3$, then

$$
\sum 3\left\lfloor\frac{i}{2}\right\rfloor x_{i}>\sum i x_{i}=\frac{3 n}{4}
$$

Thus,

$$
\alpha(\langle V \backslash X\rangle)=\sum\left\lfloor\frac{i}{2}\right\rfloor x_{i}>\frac{n}{4} .
$$

Therefore, $x_{i}=0$, for all $i>3$. Let $Y_{i} \subset V \backslash X$ such that $\left\langle Y_{i}\right\rangle \cong K_{3}$, $i=3, \ldots, \frac{n}{4}$. Let $x \in X$. As $\Gamma$ is connected and $\Gamma \not K_{4},\left\langle Y_{i} \cup\{x\}\right\rangle \not K_{4}$ and, in consequence, $\alpha\left(\left\langle Y_{i} \cup\{x\}\right\rangle\right)=2, \forall i$. Let $W=\left\{x, y_{1}, y_{2}, \ldots, y_{\frac{n}{4}}\right\}$, where $y_{i} \in Y_{i}$ and $y_{i} \nsim x$. As $W$ is an independent set, the result follows by contradiction.

It is well-known that the independent set problem is NP-complete [1]. Hence, a direct consequence of (2) is the following:

Remark 3.2. The minimum global strong offensive alliance problem is NP-complete.

There are some classes of cubic graphs in which we can compute the global strong offensive alliance number in terms of the order. For instance, if $\Gamma=$ $\mathcal{L}\left(\Gamma_{1}\right)$ is a cubic graph of order $n \geq 6$, then we have $\gamma_{\hat{0}}(\Gamma)=2 \gamma_{\hat{0}}\left(\Gamma_{1}\right)=\frac{2 n}{3}$. That is, $\Gamma_{1}=(V, E)$ is a bipartite semiregular graph of degrees 2 and 3 . If $V_{3} \subset V$ denotes the vertex-set of degree 3 in $\Gamma_{1}$, then $\gamma_{\hat{0}}\left(\Gamma_{1}\right)=\left|V_{3}\right|=\alpha(\Gamma)$. On the other hand, $\left|V_{3}\right|=\frac{n}{3}$. Therefore, $\left.\gamma_{\hat{0}}(\Gamma)=n-\alpha(\Gamma)\right)=3 \gamma_{\hat{0}}(\Gamma)-$ $\gamma_{\hat{0}}(\Gamma)=\frac{2 n}{3}$. Notice that in this class of graphs are included the cubic graphs of order $n \geq 6$ having $\frac{n}{3}$ independent triangles ${ }^{1}$.

Theorem 3.3. [7, 13] Let $\Gamma$ be a simple graph of order $n$ and minimum degree $\delta$. Let $\mu$ be the Laplacian spectral radius of $\Gamma$. Then

$$
\frac{n}{\mu}\left\lceil\frac{\delta+1}{2}\right\rceil \leq \gamma_{o}(\Gamma) \leq \frac{n(2 \mu-\delta)}{2 \mu} .
$$

Corollary 3.4. Let $\Gamma$ be a $\delta$-regular graph of order $n$. Then

$$
\frac{n}{4}\left\lceil\frac{2 \delta-1}{2}\right\rceil \leq \gamma_{o}(\mathcal{L}(\Gamma)) \leq \frac{n(\delta+1)}{4}
$$

\footnotetext{
${ }^{1} \mathrm{~A}$ set of triangles is independent if it contains no common vertices.
} 
Proof. We denote by $A$ the adjacency matrix of $\mathcal{L}(\Gamma)$ and by $2(\delta-1)=$ $\lambda_{0}>\lambda_{1}>\cdots>\lambda_{b}=-2$ its distinct eigenvalues. We denote by $L$ the Laplacian matrix of $\mathcal{L}(\Gamma)$ and by $\mu_{0}=0<\mu_{1}<\cdots<\mu_{b}$ its distinct Laplacian eigenvalues. Then, since $L=2(\delta-1) I_{n}-A$, the eigenvalues of both matrices, $A$ and $L$, are related by

$$
\mu_{l}=2(\delta-1)-\lambda_{l}, \quad l=0, \ldots, b .
$$

Thus, the Laplacian spectral radius of $\mathcal{L}(\Gamma)$ is $\mu_{b}=2 \delta$. Therefore, the result immediately follows

In the case of regular graphs of even degree all global offensive alliance is strong. Hence we have $\gamma_{o}(\Gamma)=\gamma_{\hat{o}}(\Gamma)$. Therefore, by Corollary 3.4 we obtain that if $\Gamma$ is a cubic graph, then

$$
\frac{3 n}{4} \leq \gamma_{\hat{o}}(\mathcal{L}(\Gamma))=\gamma_{o}(\mathcal{L}(\Gamma)) \leq n .
$$

The above bounds are tight. Example of equality in above upper bound is the complete graph of order 4: $\gamma_{o}\left(\mathcal{L}\left(K_{4}\right)=4=n\right.$. In the case of the bipartite complete graph $\Gamma=K_{3,3}$ we have $\gamma_{o}\left(\mathcal{L}\left(K_{3,3}\right)\right)=5$ and the above lower bound gives $\frac{9}{2} \leq \gamma_{o}\left(\mathcal{L}\left(K_{3,3}\right)\right)$.

Theorem 3.5. [13] For all graph $\Gamma$ of order $n$, minimum degree $\delta$ and maximum degree $\Delta$,

$$
\frac{2 n}{3} \geq \gamma_{0}(\Gamma) \geq \begin{cases}\left\lceil\frac{n(\delta+1)}{2 \Delta+\delta+1}\right\rceil & \text { if } \delta \text { odd; } \\ \left\lceil\frac{n \delta}{2 \Delta+\delta}\right\rceil & \text { otherwise; }\end{cases}
$$

As we show in the following result, the bound $\gamma_{o}(\Gamma) \leq \frac{2 n}{3}$ is improved for the case of regular graphs of odd degree.

Theorem 3.6. For all regular graph $\Gamma$ of order $n$ and odd degree $\delta$,

$$
\frac{n(\delta+1)}{3 \delta+1} \leq \gamma_{o}(\Gamma) \leq \frac{n}{2}
$$

Proof. The lower bound is a particular case of Theorem 3.5. Let $\{X, Y\}$ be a bipartition of $V$ such that $|X|=|Y|=\frac{n}{2}$ and the edge-cut between $X$ and $Y$ has maximum size. If neither $X$ nor $Y$ are global offensive alliances in $\Gamma$, then there exist $x \in X$ and $y \in Y$ such that $\left|N_{Y}(x)\right| \leq\left|N_{X}(x)\right|$ and $\left|N_{X}(y)\right| \leq\left|N_{Y}(y)\right|$. Since $\Gamma$ is regular of odd degree, $\left|N_{Y}(x)\right|<\left|N_{X}(x)\right|$ and $\left|N_{X}(y)\right|<\left|N_{Y}(y)\right|$. Thus, by contradiction we deduce the result. 
In the case of cubic graphs we have,

$$
\frac{2 n}{5} \leq \gamma_{o}(\Gamma) \leq \frac{n}{2}
$$

Example of equality in above upper bound is the family of cubic graphs $\Gamma=C_{r} \times K_{2}$, where $C_{r}$ denotes the $r$-cycle graph. In this case $\gamma_{o}(\Gamma)=r$.

Proposition 3.7. Let $\Gamma$ be a cubic graph of order $n$. All global offensive alliance in $\Gamma$ of cardinality $\frac{2 n}{5}$ is an independent set.

Proof. Let $X \subset V$ be a global offensive alliance in $\Gamma$ of cardinality $\frac{2 n}{5}$. Let $c$ be the size of the edge-cut between $X$ and $V \backslash X$.As $X$ is a 2-dominating set, $2 \frac{3 n}{5} \leq c$. Moreover, $c \leq 3 \frac{2 n}{5}$, so the size of $\langle X\rangle$ is zero.

A set nonempty set $S \subseteq V$ is a strong defensive alliance in $\Gamma$ if for every $v \in S, 2\left|N_{S}(v)\right| \geq \delta(v)$. A set $X \subseteq V$ is strong defensive alliance free if for all strong defensive alliance $S, S \backslash X \neq \emptyset$, i.e., $X$ do not contain any strong defensive alliance as a subset [10]. A strong defensive alliance free set $X$ is maximal if for all $v \notin X$, exists $S \subseteq X$ such that $S \cup\{v\}$ is a strong defensive alliance. A maximum strong defensive alliance free set is a maximal strong defensive alliance free set of largest cardinality. We denote by $\phi_{0}(\Gamma)$ the cardinality of a maximum strong defensive alliance free set of $\Gamma$.

Similarly, a set $Y \subseteq V$ is a strong defensive alliance cover if for all strong defensive alliance $S, S \cap Y \neq \emptyset$, i.e., $Y$ contains at least one vertex from each strong defensive alliance of $\Gamma$. A strong defensive alliance cover $Y$ is minimal if no proper subset of $Y$ is a strong defensive alliance cover. A minimum strong defensive alliance cover is a minimal cover of smallest cardinality. We will denote by $\zeta_{0}(\Gamma)$ the cardinality of a minimum strong defensive alliance cover of $\Gamma$.

Lemma 3.8. [9] If $X \subset V$ is a global offensive alliance in $\Gamma=(V, E)$, then the set $V \backslash X$ is strong defensive alliance free in $\Gamma$.

Lemma 3.9. [10] If each block ${ }^{2}$ of $\Gamma$ is an odd clique or an odd cycle, then $\phi_{0}(\Gamma) \leq \zeta_{o}(\Gamma)$.

Theorem 3.10. Let $\Gamma$ be a cubic graph of order $n$. If each block of $\Gamma$ is an odd cycle, then

$$
\gamma_{o}(\Gamma)=\frac{n}{2}
$$

\footnotetext{
${ }^{2} \mathrm{~A}$ block is a maximal 2-connected subgraph of a given graph $\Gamma$.
} 
Proof. By Lemma 3.9 and $\phi_{0}(\Gamma)+\zeta_{0}(\Gamma)=n$ we have $\phi_{0}(\Gamma) \leq \frac{n}{2}$. Moreover, by Lemma 3.8 we have $n-\gamma_{o}(\Gamma) \leq \phi_{0}(\Gamma)$. Hence, $\gamma_{o}(\Gamma) \geq \frac{n}{2}$. By Theorem 3.6 we conclude the proof.

\subsection{Independent offensive alliances}

As a consequence of Theorem 3.1 we obtain the following result.

Corollary 3.11. Let $\Gamma$ be a cubic graph. $\Gamma$ has a global strong independent offensive alliance if and only if $\Gamma$ is a bipartite graph.

Proof. In the case of cubic graphs we have $\frac{n}{2} \leq \gamma_{\hat{o}}(\Gamma)$ and $\alpha(\Gamma) \leq \frac{n}{2}$. Both equalities holds true if and only if $\Gamma$ is a bipartite graph.

In the case of a bipartite cubic graph we have,

$$
\gamma_{i}(\Gamma)=\gamma_{o}(\Gamma)=\gamma_{\hat{o}}(\Gamma)=\gamma(\Gamma)=\alpha(\Gamma)=\frac{n}{2} .
$$

For all graph having independent offensive alliances we have $\gamma_{o}(\Gamma) \leq$ $\gamma_{i}(\Gamma)$. Example of equality is the Petersen graph $O_{3}$ and the class of bipartite cubic graphs. Obviously, if $\alpha(\Gamma)<\gamma_{o}(\Gamma)$, then $\Gamma$ do not contains global independent offensive alliances. Examples of graphs having $\alpha(\Gamma)<\gamma_{o}(\Gamma)$ are the graphs isomorphic to $C_{2 k+1} \times K_{2}$. In this case $2 k=\alpha(\Gamma)<\gamma_{o}(\Gamma)=2 k+1$.

Theorem 3.12. Let $\Gamma=(V, E)$ be a cubic graph of order $n$. If $\Gamma$ has a global independent offensive alliance, then:

1. $\frac{2 n}{5} \leq \gamma_{i}(\Gamma) \leq \frac{n}{2}$.

2. $\gamma_{i}(\Gamma)=\frac{n}{2}$ if and only if $\Gamma$ is a bipartite graph.

3. $\gamma_{i}(\Gamma)=\frac{2 n}{5}$ if and only if there exists an independent set $X \subset V$ such that $\langle V \backslash X\rangle$ is a 1-factor of size $\frac{3 n}{10}$.

Proof. Let $\Gamma=(V, E)$ be a cubic graph of order $n$.

1. If $\Gamma$ has a global independent offensive alliance, then $\frac{2 n}{5} \leq \gamma_{o}(\Gamma) \leq$ $\gamma_{i}(\Gamma) \leq \alpha(\Gamma) \leq \frac{n}{2}$.

2. If $X \subset V$ is an independent set, then $V \backslash X$ is a 3-dominating set of $\Gamma$. Hence, if $|X|=\frac{n}{2}$, the edge-cut between $X$ and $V \backslash X$ has size $3 \frac{n}{2}$, so $\Gamma$ is a bipartite graph. Conversely, if $\Gamma$ is a bipartite cubic graph, then $\gamma_{i}(\Gamma)=\frac{n}{2}$. 
3. Let $X$ be a global independent offensive alliance in $\Gamma$ such that $|X|=$ $\frac{2 n}{5}$. The edge-cut between $X$ and $V \backslash X$ has size $3 \frac{2 n}{5}$. Since $|V \backslash X|=\frac{3 n}{5}$ and $X$ is a 2-dominating set, each vertex of $V \backslash X$ have one neighbor in $V \backslash X$, so $\langle V \backslash X\rangle$ is a 1 -factor of size $\frac{3 n}{10}$. The converse is immediate.

Corollary 3.13. Let $\Gamma$ be a cubic graph of order $n$. If $\alpha(\Gamma)<\frac{2 n}{5}$, then $\Gamma$ do not contains global independent offensive alliances.

Examples of graphs having $\alpha(\Gamma)<\frac{2 n}{5}$ are the complete graph, $K_{4}$, and the graph $\Gamma=K_{3} \times K_{2}$.

Theorem 3.14. Let $\Gamma$ be a cubic graph of order $n$. If $a_{i}(\Gamma)<\gamma_{i}(\Gamma)$, then

$$
\frac{n+2}{4} \leq a_{i}(\Gamma) \leq \frac{n-2}{2} .
$$

Proof. If $S \subset V$ is an independent set in $\Gamma=(V, E)$, then

$$
\frac{3 n}{2}=3|S|+\frac{\sum_{v \in V \backslash S}\left|N_{V \backslash S}(v)\right|}{2} .
$$

Moreover, if $S \subset V$ is an independent offensive alliance in $\Gamma$ such that $|S|=$ $a_{i}(\Gamma)<\gamma_{i}(\Gamma)$, then there exists at least one vertex $v \in V$ such that $v \notin$ $(S \cup \partial S)$. Hence,

$$
\frac{\sum_{v \in V \backslash S}\left|N_{V \backslash S}(v)\right|}{2} \geq 3
$$

By (6) and (7) we obtain the upper bound. On the other hand, since $S$ is an offensive alliance in $\Gamma=(V, E)$, then

$$
3|S| \geq \sum_{v \in \partial S}\left|N_{S}(v)\right| \geq \sum_{v \in \partial S}\left|N_{V \backslash S}(v)\right|+|\partial S| .
$$

Moreover, as $S$ is an independent set $|\partial S| \geq 3$. Thus,

$$
3|S|+\sum_{v \in \partial S}\left|N_{V \backslash S}(v)\right| \geq \frac{3 n}{2} .
$$

By (6) and (8) we obtain de result.

Example of equality in above bounds is the complete bipartite graph $\Gamma=K_{3,3}$. In this case $a_{i}(\Gamma)=2$. 


\section{References}

[1] M. R. Garey and D. S. Johnson. Computers and Intractability: A guide to Theory of NP-Completeness. Freeman, San Francisco, 1979.

[2] M. Chellali and T. Haynes, Global alliances and independence in trees. Submitted.

[3] M. Chellali, Offensive alliances in trees. Submitted.

[4] O. Favaron, G. Fricke, W. Goddard, S. M. Hedetniemi, S. T. Hedetniemi, P. Kristiansen, R. C. Laskar and D. R. Skaggs, Offensive alliances in graphs. Discuss. Math. Graph Theory 24 (2)(2004), 263-275.

[5] T. W. Haynes, S. T. Hedetniemi, and M. A. Henning, Global defensive alliances in graphs, Electron. J. Combin. 10 (2003), Research Paper 47.

[6] P. Kristiansen, S. M. Hedetniemi and S. T. Hedetniemi, Alliances in graphs. J. Combin. Math. Combin. Comput. 48 (2004), 157-177.

[7] J. A. Rodríguez and J. M. Sigarreta, Spectral study of alliances in graphs. Discussiones Mathematicae Graph Theory. In press.

[8] J. A. Rodríguez and J. M. Sigarreta, Global alliances in planar graphs. Submitted.

[9] J. A. Rodríguez and J. M. Sigarreta, Alliances versus cover and alliance free sets. Submitted.

[10] K. H. Shafique and R. D. Dutton, A tight bound on the cardinalities of maximum alliance-free and minimum alliance-cover sets. Submitted.

[11] K. H. Shafique and R. D. Dutton, Maximum alliance-free and minimum alliance-cover sets. Congr. Numer. 162 (2003), 139-146

[12] J. M. Sigarreta and J. A. Rodríguez, On defensive alliances and line graphs. Appl. Math. Lett. 19 (12) (2006) 1345-1350.

[13] J. M. Sigarreta and J. A. Rodríguez, On the global offensive alliance number of a graph. Submitted. 